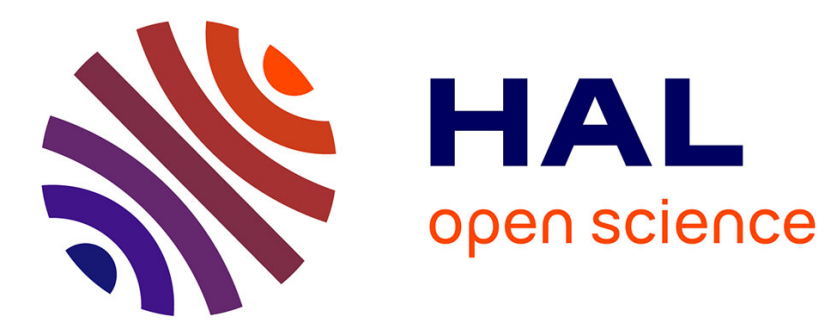

\title{
Probabilités et fluctuations quantiques
}

Michel Fliess

\section{To cite this version:}

Michel Fliess. Probabilités et fluctuations quantiques. Comptes rendus de l'Académie des sciences.

Série I, Mathématique, 2007, 344 (10), pp.663-668. 10.1016/j.crma.2007.04.001 . inria-00141719

\section{HAL Id: inria-00141719 \\ https://hal.inria.fr/inria-00141719}

Submitted on 15 Apr 2007

HAL is a multi-disciplinary open access archive for the deposit and dissemination of scientific research documents, whether they are published or not. The documents may come from teaching and research institutions in France or abroad, or from public or private research centers.
L'archive ouverte pluridisciplinaire HAL, est destinée au dépôt et à la diffusion de documents scientifiques de niveau recherche, publiés ou non, émanant des établissements d'enseignement et de recherche français ou étrangers, des laboratoires publics ou privés. 
Physique mathématique

\title{
Probabilités et fluctuations quantiques
}

\author{
Michel FLIESS a, \\ a Projet ALIEN, INRIA Futurs \\ \& Équipe MAX, LIX (CNRS, UMR 7161), École polytechnique, 91128 Palaiseau, France \\ Reçu le $* * * * * ;$ accepté après révision le +++++ \\ Présenté par $£ £ £ £ £$
}

\section{Résumé}

Cette Note esquisse une construction mathématique simple et naturelle du caractère probabiliste de la mécanique quantique. Elle utilise l'analyse non standard et repose sur l'interprétation due à Feynman, mise en avant dans certaines approches fractales, du principe d'incertitude de Heisenberg, c'est-à-dire des fluctuations quantiques. On aboutit ainsi à des équations différentielles stochastiques, comme dans la mécanique stochastique de Nelson, découlant de marches aléatoires infinitésimales. Pour citer cet article : M. Fliess, C. R. Acad. Sci. Paris, Ser. I 344 (2007).

\begin{abstract}
Probabilities and quantum fluctuations. This Note is sketching a simple and natural mathematical construction for explaining the probabilistic nature of quantum mechanics. It employs nonstandard analysis and is based on Feynman's interpretation of the Heisenberg uncertainty principle, i.e., of the quantum fluctuations, which was brought to the forefront in some fractal approaches. It results, as in Nelson's stochastic mechanics, in stochastic differential equations which are deduced from infinitesimal random walks. To cite this article: M. Fliess, C. R. Acad. Sci. Paris, Ser. I 344 (2007).
\end{abstract}

\section{Abridged English version}

We are sketching a "simple and natural" explanation of the probabilistic nature of quantum mechanics. This is achieved by utilizing the mathematical formalism of nonstandard analysis and Feynman's interpretation of the Heisenberg uncertainty principle, i.e., of the quantum fluctuations.

\footnotetext{
Email address: Michel.Fliess@polytechnique.edu (Michel FLIESS).
} 
Non-technical presentation of the main ideas. This summary is intended for readers who are not familiar with non-standard analysis. As often in nonstandard analysis (see, e.g., [1,26]), we replace a continuous time interval by an infinite "discrete" set of infinitely closed time instants. Substituting $m \frac{\Delta x}{\Delta t}$ to $\Delta p$ in the well known expression of the uncertainty principle, where $x$ is the position, $m$ the mass, $p$ the momentum, $h=2 \pi \hbar$ the Planck constant, yields equation (1). We rewrite it by postulating that the quantity (2), where $\delta t>0$ is a given infinitesimal, is limited and appreciable, i.e., it is neither infinitely large nor infinitely small. Those computations are stemming from Feynman's interpretation [13,14] of the uncertainty principle (see, also, $[21,27]$ ): The "quantum trajectories" are fractal curves, of Hausdorff dimension 2. "Weak" mathematical assumptions permit to derive the infinitesimal difference equation (3). The lack of any further physical assumption yields the equiprobability of +1 and -1 . If $x$ is Markov, i.e., if $b$ and $\sigma$ are are functions of $t$ and $x(t)$, and not of $\{x(\tau) \mid 0 \leq \tau<t\}$, the corresponding infinitesimal random walk is "equivalent" to a stochastic differential equation in the usual sense (see, e.g., [1,5]).

Remark 1 More or less analogous random walks have already been introduced in the literature (see, e.g., [3,19,28,29]), but in another context.

Non-standard analysis. Replace the interval $[0,1]$ by the set $\mathfrak{Q}=\left\{k \delta t \mid 0 \leq k \leq N_{q}\right\}, \delta t=\frac{1}{N_{q}}$, where $N_{q}$ is an unlimited integer. A function $x: \mathfrak{Q} \rightarrow \mathbb{R}$ is said to verify the Heisenberg condition if, and only if, for any $t \in \mathfrak{Q} \backslash\{1\}$, equation (4) is satisfied, where $\sigma_{t}^{\star} \in \mathbb{R}, \sigma_{t}^{\star}>0$, is limited and appreciable. The lack of any further physical assumption leads us to postulate the following properties for $\varepsilon(t)$ in equation (5): the random variables $\varepsilon(t), t \in \mathfrak{Q} \backslash\{1\}$, are independent; $\operatorname{Pr}(\varepsilon(t)=+1)=\operatorname{Pr}(\varepsilon(t)=-1)=\frac{1}{2}$. The next properties are "natural" for the stochastic processes $\varepsilon$ and $x$ (see [26] and [4,5]): the random variables $x(0)$ and $\varepsilon(t), t \in \mathfrak{Q}$, are independent; $x(t), t>0$, is function of $\{\varepsilon(\tau) \mid 0 \leq \tau \leq t-\delta t\}$. Set $E_{t}^{\varepsilon}(\bullet)=E_{t}(\bullet \mid \varepsilon(0), \varepsilon(\delta t), \ldots, \varepsilon(t-\delta t))$. Equation (6) yields the decomposition (7) [26], where $\eta$ is a stochastic process, such that $E_{t}^{\varepsilon}(\eta(t))=0, E_{t}^{\varepsilon}\left((\eta(t))^{2}\right)=1$. Then, for all $t \in \mathfrak{Q} \backslash\{1\}, \sigma_{t}^{\star} \simeq s_{t}^{\star}, \varepsilon(t)=\eta(t)$.

We will not make any distinction between two equations of type (7) if the coefficients are limited and infinitely closed. The process $x$ is said to be Markov, or to satisfy the Markov condition, if, and only if, there exist $b \simeq D^{\varepsilon} x(t), \sigma \simeq s_{t}^{\star}$, such that they are functions of $t$ et $x(t)$, and not of $\{x(\tau) \mid 0 \leq \tau<t\}$. Assume that $x$ is Markov. Consider the infinitesimal random walk (8). According to [4] (see, also, [5]) it defines a diffusion if, and only if, the following conditions are satisfied (see [1] for another approach): $b$ and $\sigma$ are of class $S^{0}$; the shadows $\tilde{b}$ and $\tilde{\sigma}$ of $b$ et $\sigma$ are of class $C^{\infty}$; the function $\tilde{\sigma}$ is always strictly positive; the random variable $x(0)$ is almost surely limited.

\section{Introduction}

Le caractère probabiliste du quantique, confirmé par tant d'expériences, procède, depuis Born, Bohr et l'école dite de Copenhague-Göttingen, d'une axiomatique de la fonction d'onde de Schrödinger, qui, comme on le sait, a fait naître, à cause de son étrangeté et de ses apparents paradoxes, d'innombrables commentaires (cf. [6]). Cette Note (voir [17] pour une version préliminaire) vise à déduire cette nature aléatoire de considérations mathématiques «simples et naturelles ». Elle repose sur

- l'interprétation par Feynman [13,14] du principe d'incertitude de Heisenberg, c'est-à-dire des fluctuations quantiques ;

- l'analyse non standard de Robinson [33], déjà employée en quantique (voir, par exemple, [1], sa bibliographie, et $[19,27]$ ), et, plus précisément,

- une discrétisation infinitésimale du temps,

- le calcul non standard des probabilités de Nelson [26] et son extension aux équations différentielles stochastiques $[4,5]$. 
On aboutit à des équations différentielles stochastiques grâce à de marches aléatoires infinitésimales. Nous empruntons ainsi à des degrés divers à trois alternatives aux fondements du quantique, parfois très controversées, dont les liens avaient déjà été constatés dans la littérature (cf. [3]) : l'approche fractale, la mécanique stochastique et les marches aléatoires.

(i) Nous suivons la première, portée par Nottale [27] (voir aussi [21]), dans sa mise en valeur de l'interprétation due à Feynman du principe d'incertitude, qui semblait plus ou moins oubliée.

(ii) Les équations différentielles stochastiques ordinaires sont un pivot de la seconde, inventée par Fényes [12], développée et popularisée par Nelson [22,23,25]. Cette mécanique continue à susciter une littérature abondante (cf. $[7,11,18,30,31,32,34,35]$ ), en dépit de critiques virulentes (cf. [8,18]). Voir, par exemple, $[2,8]$ pour une comparaison avec la mécanique quantique euclidienne, que beaucoup, Nelson y compris, jugent plus convaincante.

(iii) Renvoyons pour la troisième aux fameux échiqier (chessboard) de Feynman [14], aux travaux d'Ord (cf. $[28,29])$ et à $[3,19]$.

Remarque 1 On se restreint à un espace de dimension 1 afin d'alléger l'écriture.

Remarque 2 Alors que certains travaux autour de la mécanique stochastique se rattachent à l'automatique et, plus précisément, à la commande optimale stochastique (cf. [20]), notre point de vue fait suite à une approche nouvelle [16] de l'estimation et de l'identification, en automatique et signal. Rappelons les liens entre le quantique actuel et certains aspects du traitement de signal (cf. [15]).

Remarque 3 On s'efforcera de dégager les rapports avec des travaux actuels sur le plongement stochastique $[9,10]$.

\section{Présentation générale}

Voici une synthèse des principales idées directrices, sans prétention de rigueur, mais, espérons-le, claire et transparente, destinée aux lecteurs peu au fait de l'analyse non standard :

(i) On remplace, comme souvent en non-standard (cf. [1,26]), le temps continu par un ensemble infini « discret » d'instants infiniment proches.

(ii) Les calculs suivants émanent d'une reformulation du principe d'incertitude, due à Feynman [13,14] (voir, aussi, [21,27]) : la «trajectoire quantique » est une courbe continue, non dérivable, c'est-à-dire fractale, de dimension de Hausdorff 2, traduisant la «Zitterbewegung », c'est-à-dire les fluctuations quantiques. Soient $x$ la position, $m$ la masse, $p$ la quantité de mouvement, $h=2 \pi \hbar$ la constante de Planck. Le symbole $\Delta$ désigne un «petit » accroissement; ainsi $\Delta x=x(t+\Delta t)-x(t)$ et, donc, $\Delta p \simeq m \frac{\Delta x}{\Delta t}$. L'expression familière $\Delta x \Delta p \gtrsim \hbar$ du principe d'incertitude devient (cf. [21], p. 85) :

$$
\frac{(\Delta x)^{2}}{\Delta t} \gtrsim \frac{\hbar}{m}
$$

Soit $\delta t>0$ infinitésimal donné. On réécrit (1) en postulant que

$$
\frac{(x(t+\delta t)-x(t))^{2}}{\delta t}
$$

est limité et appréciable, c'est-à-dire ni infiniment grand ni infiniment petit.

Remarque 4 Voir, à propos de l'irréversibilité thermodynamique, [3] pour une condition voisine de (1)-(2) dans un espace-temps discret. 
(iii) On en déduit, moyennant des hypothèses mathématiques «faibles », l'équation aux différences infinitésimales

$$
x(t+\delta t)=x(t)+b \delta t \pm \sigma \sqrt{\delta t}
$$

(iv) L'absence de toute hypothèse physique supplémentaire conduit à postuler l'équiprobabilité de \pm 1 .

(v) Si $x$ est markovien, c'est-à-dire si $b$ et $\sigma$ sont fonctions de $t$ et $x(t)$, et non de $\{x(\tau) \mid 0 \leq \tau<t\}$, la marche aléatoire infinitésimale définie par (3) « équivaut » à une équation différentielle stochastique au sens usuel (cf. $[1,4,5])$, comme en mécanique stochastique.

\section{Analyse non standard}

On rédige selon la terminologie $I S T$ de $[24,26]$.

\subsection{Condition de Heisenberg}

Remplaçons l'intervalle $[0,1]$ par $\mathfrak{Q}=\left\{k \delta t \mid 0 \leq k \leq N_{q}\right\}, \delta t=\frac{1}{N_{q}}$, où $N_{q}$ est un entier illimité. Appelons $\mathfrak{Q}$ et $\delta t$, respectivement, l'échelle et le pas de temps quantiques. Une fonction $x: \mathfrak{Q} \rightarrow \mathbb{R}$ est dite vérifier la condition de Heisenberg si, et seulement si, pour tout $t \in \mathfrak{Q} \backslash\{1\}$,

$$
\frac{(x(t+\delta t)-x(t))^{2}}{\delta t} \simeq\left(\sigma_{t}^{\star}\right)^{2}
$$

où $\sigma_{t}^{\star} \in \mathbb{R}, \sigma_{t}^{\star}>0$, est limité et appréciable. Il en découle

$$
x(t+\delta t)-x(t) \simeq \varepsilon(t)\left(\sigma_{t}^{\star}\right) \sqrt{\delta t} \text { où } \varepsilon(t)= \pm 1
$$

\section{2. Équiprobabilité de +1 et -1}

Postulons les deux propriétés suivantes, qui découlent «naturellement » de l'absence de toute hypothèse physique supplémentaire :

- les $\varepsilon(t), t \in \mathfrak{Q} \backslash\{1\}$, sont des variables aléatoires indépendantes,

$-\operatorname{Pr}(\varepsilon(t)=+1)=\operatorname{Pr}(\varepsilon(t)=-1)=\frac{1}{2}$.

\subsection{Processus stochastiques}

Comme $\varepsilon$ peut être vu comme un processus stochastique, il est loisible de postuler les propriétés suivantes (cf. [26] et $[4,5])$ :

$-x(t)$ est un processus stochastique;

- les variables aléatoires $x(0)$ et $\varepsilon(t), t \in \mathfrak{Q}$, sont indépendantes;

$-x(t), t>0$, est fonction de $\{\varepsilon(\tau) \mid 0 \leq \tau \leq t-\delta t\}$.

Notons $E_{t}^{\varepsilon}(\bullet)$ l'espérance conditionnelle du processus • en l'instant $t \in \mathfrak{Q}$, sachant le passé du processus $\varepsilon$. Posons

$$
\begin{gathered}
D^{\varepsilon} x(t)=\frac{E_{t}^{\varepsilon}(x(t+\delta t)-x(t))}{\delta t} \\
s_{t}^{\star} \simeq \sqrt{\frac{\left(x(t+\delta t)-x(t)-D^{\varepsilon} x(t) \delta t\right)^{2}}{\delta t}}
\end{gathered}
$$


Supposons $D^{\varepsilon} x(t)$ limité. On obtient la décomposition [26] (voir, aussi, [4]) :

$$
x(t+\delta t)=x(t)+\left(D^{\varepsilon} x(t)\right) \delta t+\left(s_{t}^{\star}\right) \eta(t) \sqrt{\delta t}
$$

où $\eta$ est un processus stochastique, tel que $E_{t}^{\varepsilon}(\eta(t))=0, E_{t}^{\varepsilon}\left((\eta(t))^{2}\right)=1$. La proposition suivante relie les quantités $\sigma_{t}^{\star}$ et $\varepsilon(t)$ des paragraphes précédents aux nouvelles :

Proposition 3.1 Pour tout $t \in \mathfrak{Q} \backslash\{1\}$, il est loisible de poser $\sigma_{t}^{\star} \simeq s_{t}^{\star}, \varepsilon(t)=\eta(t)$.

\subsection{Condition de Markov}

Soit

$$
x_{i}(t+\delta t)=x_{i}(t)+\left(D^{\varepsilon} x_{i}(t)\right) \delta t+\left(s_{i, t}^{\star}\right) \varepsilon(t) \sqrt{\delta t}
$$

où $i=1,2$. La propriété suivante est facile :

Lemme 3.2 Si, pour tout $t \in \mathfrak{Q} \backslash\{1\}$, les quantités infiniment proches $D^{\varepsilon} x_{1}(t) \simeq D^{\varepsilon} x_{2}(t), s_{1, t}^{\star} \simeq s_{2, t}^{\star}$, $x_{1}(0) \simeq x_{2}(0)$ sont limitées en valeur absolue par $C \in \mathbb{R}$, la différence $x_{1}(t)-x_{2}(t)$ reste infinitésimale pour tout $t \in \mathfrak{Q}$.

Nous ne distinguerons pas deux équations de type (7) avec coefficients limités et infiniment proches. On dit que $x$ est markovien, ou satisfait la condition de Markov, si, et seulement si, il existe des quantités $b \simeq D^{\varepsilon} x(t), \sigma \simeq s_{t}^{\star}$ fonctions de $t$ et $x(t)$, et non de $\{x(\tau) \mid 0 \leq \tau<t\}$.

\subsection{Diffusions}

Supposons, dorénavant, $x$ markovien et considérons la marche aléatoire infinitésimale

$$
x(t+\delta t)=x(t)+b(t, x(t)) \delta t+\sigma(t, x(t)) \varepsilon(t) \sqrt{\delta t}
$$

Renvoyons à [26] (voir, aussi, [4,5]) pour la notion d'équivalence de processus stochastiques. Le représentant (8) définit, selon [4] (voir, aussi, [5]), une diffusion si, et seulement si, les conditions suivantes sont satisfaites :

- $b$ et $\sigma$ sont de classe $S^{0}$,

- les ombres $\tilde{b}$ et $\tilde{\sigma}$ de $b$ et $\sigma$ sont de classe $C^{\infty}$,

- la fonction $\tilde{\sigma}$ est toujours strictement positive,

- la variable aléatoire $x(0)$ est presque sûrement limitée.

Remarque 5 Voir [1] pour une autre approche des liens entre (8) et équations différentielles stochastiques, où les conditions requises pour $b$ et $\sigma$ sont sensiblement différentes.

Remerciements. L'auteur exprime sa reconnaissace à E. Benoît (La Rochelle) pour lui avoir communiqué la référence [4], à J.-M. Lévy-Leblond (Nice) pour des remises en question salutaires, à P. Rouchon (Paris) pour des discussions précieuses, et à T. Sari (Mulhouse) pour des conseils utiles.

\section{Références}

[1] S. Albeverio, J.E. Fenstad, R. Hoegh-Krøhn, T. Lindstrøm, Nonstandard Methods in Stochastic Analysis and Mathematical Physics, Academic Press, Orlando, FL, 1986.

[2] S. Albeverio, K. Yasue, J.C. Zambrini, Euclidean quantum mechanics: analytical approach, Ann. Inst. H. Poincaré Phys. théor., 50, 1989, 259-308. 
[3] J.-P. Badiali, Entropy, time-irreversibility and the Schrödinger equation in a primarily discrete spacetime, J. Phys. A: Math. Gen., 38, 2005, 2835-2847.

[4] E. Benoît, Diffusions discrètes et mécanique stochastique, Prépubli. Lab. Math. J. Dieudonné, Université de Nice, 1989.

[5] E. Benoît, Random walks and stochastic differential equations, in: F. \& M. Diener (Eds), Nonstandard Analysis in Practice, Springer, Berlin, 1995, p. 71-90.

[6] M. Bitbol, L'aveuglante proximité du réel, Flammarion, Paris, 1998.

[7] P. Blanchard, P. Combe, W. Zheng, Mathematical and physical aspects of stochastic mechanics, Lect. Notes Physics, vol. 281, Springer, Berlin, 1987.

[8] K.L. Chung, J.C. Zambrini, Introduction to Random Time and Quantum Randomness, World Scientific, Singapour, 2003.

[9] J. Cresson, S. Darses, Plongement stochastique des systèmes lagrangiens, C.R. Acad. Sci. Paris Ser. I, 342, 2006, 333-336.

[10] J. Cresson, S. Darses, Théorème de Noether stochastique, C.R. Acad. Sci. Paris Ser. I, 344, 2007, 259-264.

[11] M Davidson, Stochastic mechanics, trace dynamics, and differential space - a synthesis, Prépublication, 2006 (accessible sur http://www.arxiv.org/abs/quant-ph/0602211).

[12] I. Fényes, Eine wahrscheinlichkeitstheoretische Begründung und Interpretation der Quantenmechanik, Zeitschrift Physik, 132, 1952, 81-106.

[13] R.P. Feynman, Space-time approach to non-relativistic quantum mechanics, Rev. Modern Physics, 22, 1948, 367-387.

[14] R.P. Feynman, A.R. Hibbs, Quantum Mechanics and Path Integrals, McGraw-Hill, New York, 1965.

[15] P. Flandrin, Temps-fréquence, $2^{e}$ éd., Hermès, Paris, 1998.

[16] M. Fliess, Analyse non standard du bruit, C.R. Acad. Sci. Paris Ser. I, 342, 2006, 797-802.

[17] M. Fliess, Une approche intrinsèque des fluctuations quantiques en mécanique stochastique, Manuscrit, 2006 (accessible sur http://hal.inria.fr/inria-00118460).

[18] L. Fritsche, M. Haugk, A new look at the derivation of the Schrödinger equation from Newtonian mechanics, Annalen Physik, 12, 2003, 371-403.

[19] S. Gudder, Hyperfinite quantum random walks, Chaos Solitons Fractals, 7, 1996, 669-679

[20] F. Guerra, L.M. Morato, Quantization of dynamical systems and stochastic control theory, Physical Rev. D, 27, 1983, 1774-1786

[21] H. Kröger, Fractal geometry in quantum mechanics, field theory and spin systems, Physics Rep., 323, 2000, 81-181.

[22] E. Nelson, Derivation of the Schrödinger equation from Newtonian mechanics, Physical Rev., 150, 1966, 1079-1085.

[23] E. Nelson, Dynamical Theories of Brownian Motion, Princeton University Press, Princeton, NJ, 1967 (2e éd., datant de 2001, accessible sur http://www . math.princeton.edu/\%7Enelson/books/bmotion.pdf).

[24] E. Nelson, Internal set theory, Bull. Amer. Math. Soc., 83, 1977, 1165-1198.

[25] E. Nelson, Quantum Fluctuations, Princeton University Press, Princeton, NJ, 1985 (accessible sur http://www.math.princeton.edu/\%7Enelson/books/qf.pdf).

[26] E. Nelson, Radically Elementary Probability Theory, Princeton University Press, Princeton, NJ, 1987 (accessible sur http://www. math. princeton. edu/\%7Enelson/books/rept.pdf).

[27] L. Nottale, Fractal Space-Time and Microphysics, World Scientific, Singapour, 1993.

[28] G.N. Ord, A.S. Deakin, Random walks, continuum limits, and Schrödinger's equation, Physical Rev. A, 54, 1996, $3772-3778$

[29] G.N. Ord, R.B. Mann, Entwined pairs and Schrödinger's equation, Annals Physics, 308, 2003, 478-492.

[30] O. Oron, L.P. Horwitz, Relativistic Brownian motion and gravity as an eikonal approximation to a quantum evolution equation, Foundat. Physics, 35, 2005, 1181-1203.

[31] M. Pavon, Stochastic mechanics and the Feynman integral, J. Math. Physics, 41, 2000, 6060-6078.

[32] L. de la Peña, A.M. Cetto, The Quantum Dice: An Introduction to Stochastic Electrodynamics, Kluwer, Dordrecht, 1996.

[33] A. Robinson, Non-Standard Analysis, $2^{\text {nd }}$ ed., North-Holland, Amsterdam, 1974.

[34] L. Smolin, Matrix models and non-local hidden variables theories, in: A. Elitzur, S. Dolev, N. Kolenda (Eds), Quo Vadis Quantum Mechanics, Springer, Berlin, 2005, pp. 121-152.

[35] L. Smolin, Could quantum mechanics be an approximation to another theory?, Prépublivation, 2006 (accessible sur http://www.arxiv.org/abs/quant-ph/0609109). 